CASE REPORT

\title{
Immature Ovarian Teratoma
}

\author{
Emilija Petanovska Kostova ${ }^{1}$, Valentina Sotirovska ${ }^{2}$, Daniela Stojanovska ${ }^{3}$, Irina Prodanova ${ }^{4}$, Zorancho Petanovski ${ }^{5}$
}

\begin{abstract}
We present our experience of diagnosing immature ovarian teratoma with 2D and 3D vaginal ultrasound. A 29-year-old female patient with no symptoms was examined for routine gynecology examination before the start of an in vitro fertilization program. The patient had two previous surgeries both for ovarian cystectomy and histopathology finding of mature cystic ovarian teratoma. Left oophorectomy was performed for complex ovarian mass that demonstrated immature ovarian teratoma on final pathology. The neoplasm was well-differentiating (low-grade), according to two-tiered (low- and high-grade) system, and in IA stage, according to the Union for International Cancer Control (UICC) and American Joint Committee on Cancer (AJCC). The oncologist's council proposed that no treatment is needed.
\end{abstract}

Keywords: 2D/3D ultrasonography, Immature teratoma, Ovarian teratoma.

Donald School Journal of Ultrasound in Obstetrics and Gynecology (2020): 10.5005/jp-journals-10009-1677

\section{INTRODUCTION}

Teratomas are tumors belonging to the class of germ cell tumors as a result of abnormal differentiation of pluripotent cells. Ovarian teratomas include mature cystic teratomas (dermoid cysts), immature teratomas, and monodermal teratomas (strumaovarii, carcinoid tumors, neural tumors). Like mature cystic teratomas, immature teratomas are composed of tissues derived from the three germ layers, and they demonstrate clinically malignant behavior, they are less common ( $<1 \%$ of all ovarian teratomas) and they affect the younger age group with histologically presentation of immature tissue most frequently with neural differentiation. ${ }^{1}$

The diagnosis is made with a combination of clinical, radiological, and laboratory findings. Prognostic factors like older age at diagnosis, higher stage, and grade have worse prognosis but overall improved after chemotherapy with $72 \%$ survival for stage IV disease. ${ }^{2}$ Prognosis and treatment depend on tumor grade (a most important risk factor for relapse), stage, and karyotype. It is postulated that in ovarian immature teratoma normal $46, \mathrm{XX}$ karyotype is an indicator of favorable prognosis, whereas deviations in karyotype suggest a possibility of poor prognosis. ${ }^{3}$

The grading system is by Thurlbeck and Scully and Norris et al. ${ }^{4}$ for pure immature teratomas analyzing and describing the differentiation of the cellular elements in the tumor. ${ }^{4}$ Staging is defined the same as other ovarian cancers that are FIGO classification, describing the extent of the tumor, presence, or absence of metastasis to lymph nodes, and presence or absence of distant localization metastasis. ${ }^{5}$

\section{Case Description}

A 29-year-old female patient had attended the clinic seeking fertility treatment. The patient was unable to conceive for 3 years having active married life. A detailed history was taken and the patient has regular menstrual cycles. She had no chronic diseases and therapy. Blood group O positive and BMI 32.4. She had previous surgeries, the first surgery was before 14 years where the right adnexa was removed because of a "Dermoid" cyst that had been torqued, and the second surgery was 4 years ago laparoscopic cystectomy on the left ovary with histopathology findings of ovarian benign cystic teratoma. As for the fertility problem the couple had all the necessary examinations like semen analysis, hormonal status, 1-3/n Vitro Fertilization Centre, First Private General Hospital ReMedika, Skopje, North Macedonia; University GoceDelchev, Shtip, North Macedonia

${ }^{4} \mathrm{PHI}$ Histolab, Diagnostic Laboratory for Cytology and Histopathology, Skopje, North Macedonia

${ }^{5}$ Medical Faculty, University GoceDelchev, Shtip, North Macedonia

Corresponding Author: Emilija Petanovska Kostova, In Vitro Fertilization Centre, First Private General Hospital - ReMedika, Skopje, North Macedonia; University GoceDelchev, Shtip, North Macedonia, Phone: +389-70-750-92, e-mail: petanovskaemilij@yahoo.com

How to cite this article: Petanovska Kostova E, Sotirovska V, Stojanovska D, et al. Immature Ovarian Teratoma. Donald School J Ultrasound Obstet Gynecol 2020;14(4):359-363.

Source of support: Nil

Conflict of interest: None

PAP smear, microbiology scrubs, and hysterosalpingography. A gynecology examination was made before the start of the in vitro fertilization program. On bimanual examination, uterus and the adnexa were painless with normal dimension and localization.

On vaginal ultrasound, the uterus was with anteverted localization with normal morphology and size. Transvaginal ultrasonography showed a mass with a diameter $<4 \mathrm{~cm}$, originating from the left ovary. Based on IOTA criteria, ${ }^{6}$ the mass has been described as a unilocular-solid cyst, with one solid projection, hyperechoic in texture with small round hypoechoic/anechoic areas. The solid part, covering $0.5 \times 0.3 \times 0.6 \mathrm{~cm}$ arising from one side of the cystic wall, showed irregular form and blood supply with $\mathrm{Ri}=0.25$. There were no additional abnormal findings on the ovary, which shows a normal structure. Based on the above reported ultrasonographic data and regarding IOTA simple rules, ${ }^{7}$ the ovarian lesion was characterized as a "probably malignant tumor". There were neither indications for ascites in the intra-abdominal area nor any abnormal effusion in the Douglas pouch (Figs $1 \mathrm{~A}$ to $\mathrm{C}$ ).

Laboratory results showed that: $\mathrm{H} 4, \mathrm{Ca}-125-\mathrm{ROMA}$ index (ovarian malignancy risk algorithm) showed low risk as well as serum levels of alpha-fetoprotein (AFP), carcinoembryonic antigen (CEA), and $\beta$-subunit of chorion human gonadotropin (beta-hCG) with dilution have fluctuated in normal ranges. MRI of the upper 
and lower abdomen was also performed, as well as chest X-ray and ultrasonographic breast examination, all showing normal results.

Having analyzed the available medical information and taken into consideration the patient's previous oophorectomy, we recommended IVF, followed by operative treatment, which would have included the removal of the remaining ovary. The patient asked for a second opinion. The patient then returned to our department to accept our proposal. The research findings were quite interesting. Namely, the transvaginal ultrasound showed a dramatic increase of the cyst's solid tissue, spanning $3.6 \times 3.5 \times 4 \mathrm{~cm}$, changes in the upper, hyperechogenic foci inside and outside the solid tissue, rich vascularity (color score of IV) of the solid projection connected to the walls of the cyst with a tract resembling the solid tissue. In addition, two blood vessels feeding the solid tissue have been detected (Figs 2A to D).

The patient entered the IVF-ICSI program. A modified protocol of controlled ovarian stimulation was conducted. The obtained ovarian cells from the follicular aspiration were fertilized via the ICSi method, and a total of nine embryos in the blastocyst stage were cryopreserved for further use. Not long after the IVF procedure, a laparotomy was performed with the following findings: there were no findings of intra-abdominal adhesion nor any change in the
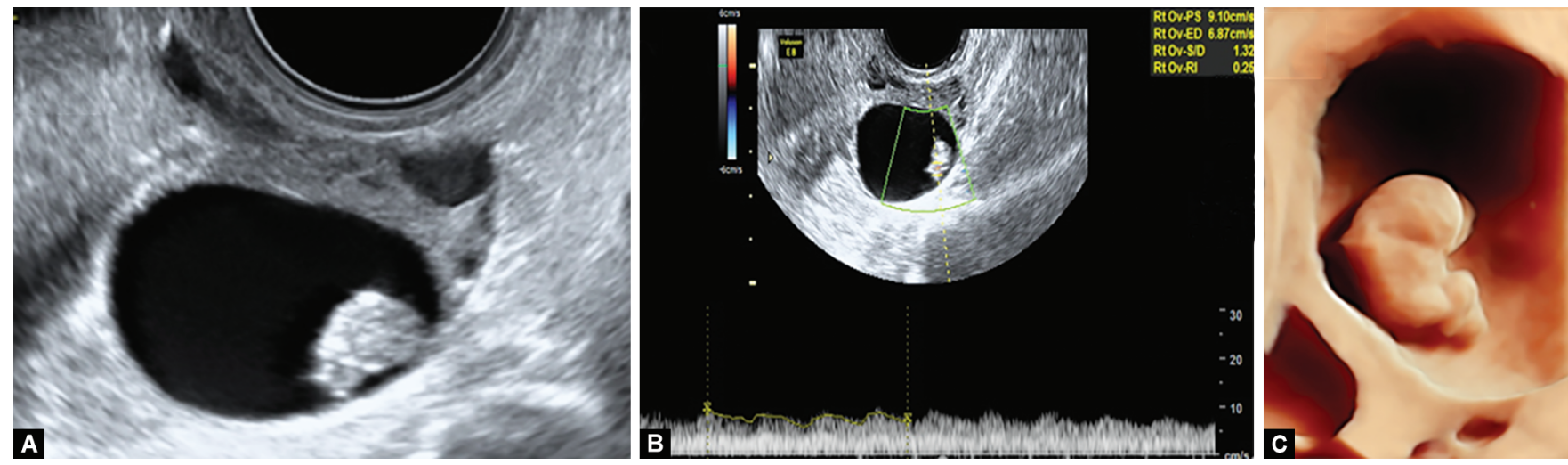

Figs 1A to C: (A) 2D ultrasound of immature teratoma. Unilocular-solid cyst, with one solid projection, hyperechoic in texture with small round hypoechoic/anechoic areas; (B) 2D ultrasound of immature teratoma: blood supply of the solid structure detected with $\mathrm{Ri}=0.25$; (C) $3 \mathrm{D}$ ultrasound of immature teratoma: clearly showing the outline of the solid tissue and its connection with the cystic wall
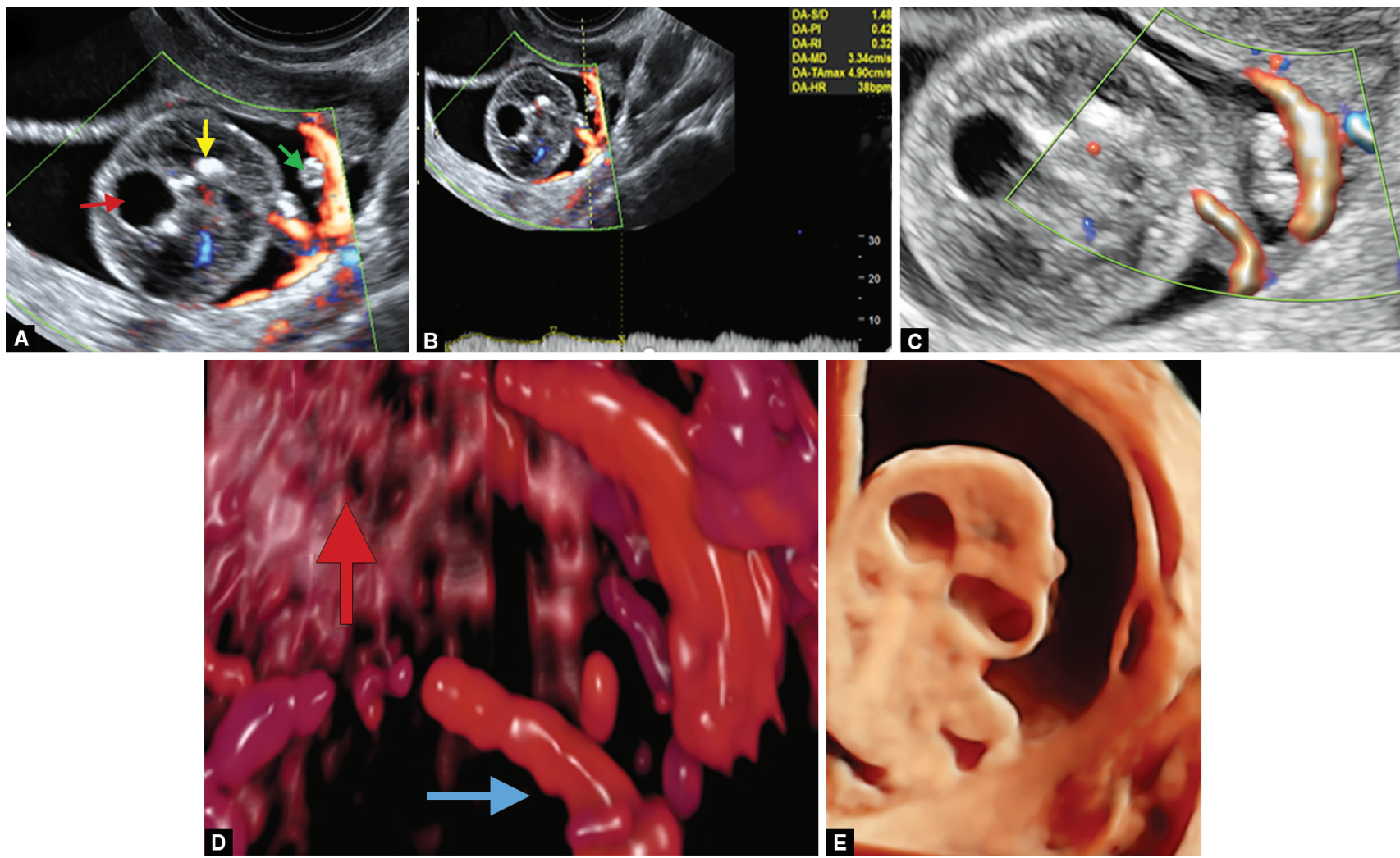

Figs 2A to E: (A) 2D ultrasound of the immature teratoma. Unilocular-solid cyst. Intracavitary solid mass with hyperechogenic pattern, cystic changes in the upper part (red arrow); hyperechogenic foci on the side (yellow arrow), and outside of the solid projection (green arrow); (B) Low $\mathrm{Rl}$ of the blood vessels of the solid mass; (C) Richly vascularized strip; (D) 3D color Doppler ultrasound image of blood vessels. Richly vascularized solid mass (red arrow) as well as the vascularization of the solid strip (blue arrow); (E) The 3D shows a clear shape of the solid mass that looked like a "fetus figure" with several clear cystic changes in the upper part 
parietal peritoneum and omentum. The uterus was of normal size, both fallopian tubes smooth, and the serosa without any pathology. Left ovary with enlarged dimensions but smooth surface. Left adnexectomy, right salpingectomy, and omentum resection were performed, and aspirate was taken for cytological analysis.

\section{Surgery and Histopathological Report Gross Findings}

An ovarian cyst measured $5 \times 5 \times 5 \mathrm{~cm}$, with an attached fallopian tube and with a smooth and shiny external surface. On cut-section cyst was unilocular, containing serous fluid, with a solitary solid component arising from the cyst wall. The solid component was soft, fleshy, and gelatinous, with coarse calcifications (Fig. 3).

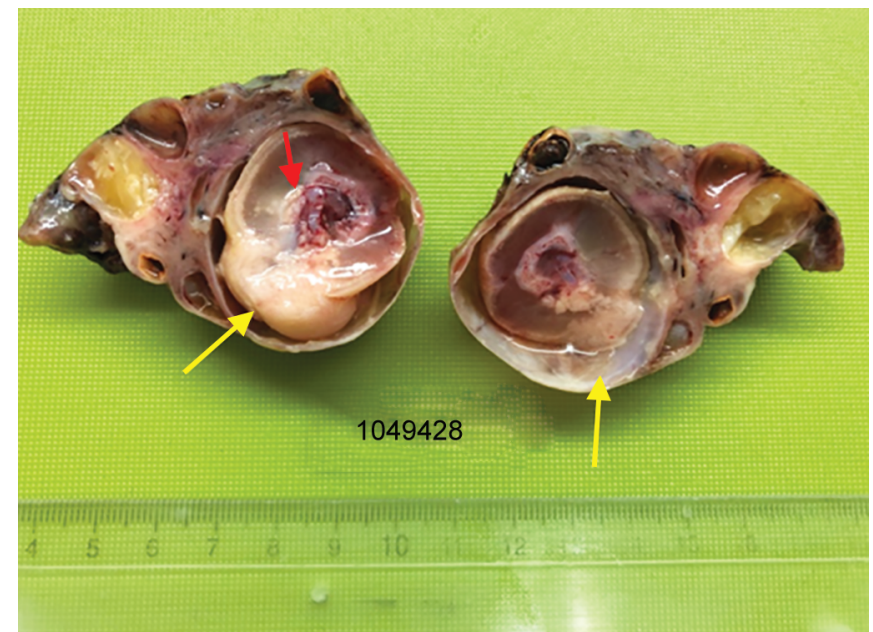

Fig. 3: Left ovary with cystic formation: immature teratoma (yellow arrow)

\section{Microscopic Findings}

\section{Mature Tissues}

Ovarian tumor reveals tissues from three germ cell layers with an admixture of predominantly mature and partly immature tissues. Mature tissues were represented with skin and skin appendages, adipose tissue, cartilage and bone, cerebral cortex with choroid plexus, and gastrointestinal epithelium (Fig. 4).

\section{Immature Tissues}

Immature tissues were represented with immature epithelium and mesenchyme (Fig. 6) and neuroectodermal tubules (Figs 5 and 6).

\section{Summary of Pathohistology Findings}

The tumor was ovarian immature teratoma with rare foci of immature neuroepithelial tissue that occupy less than 1 low power field in one slide. According to the two-stage gradation system of Crum et al., ${ }^{8}$ the neoplasm was well differentiated (low-grade) and in IA stage, according The Union for International Cancer Control (UICC) and American Joint Committee on Cancer (AJCC). The oncologist's council proposed that no treatment is needed. Cytogenetic analysis of the resected immature teratoma had revealed a 46, XX genotype. Based on the above-reported data, the oncologist's council proposed that this patient was affected by stage IA, ovarian immature teratoma and further no treatment is needed. The patient will be closely monitored.

\section{Discussion}

Ovarian immature teratomas are a rare type of malignant germ cell tumor with $<1 \%$ of all ovarian malignant tumors diagnosed. If the tumor is small symptoms may be minimal but there can be symptoms like abdominal pain and discomfort, rapidly growing abdominal mass, bloating, irregular menstrual periods, constipation, and leg weakness. Compared with mature
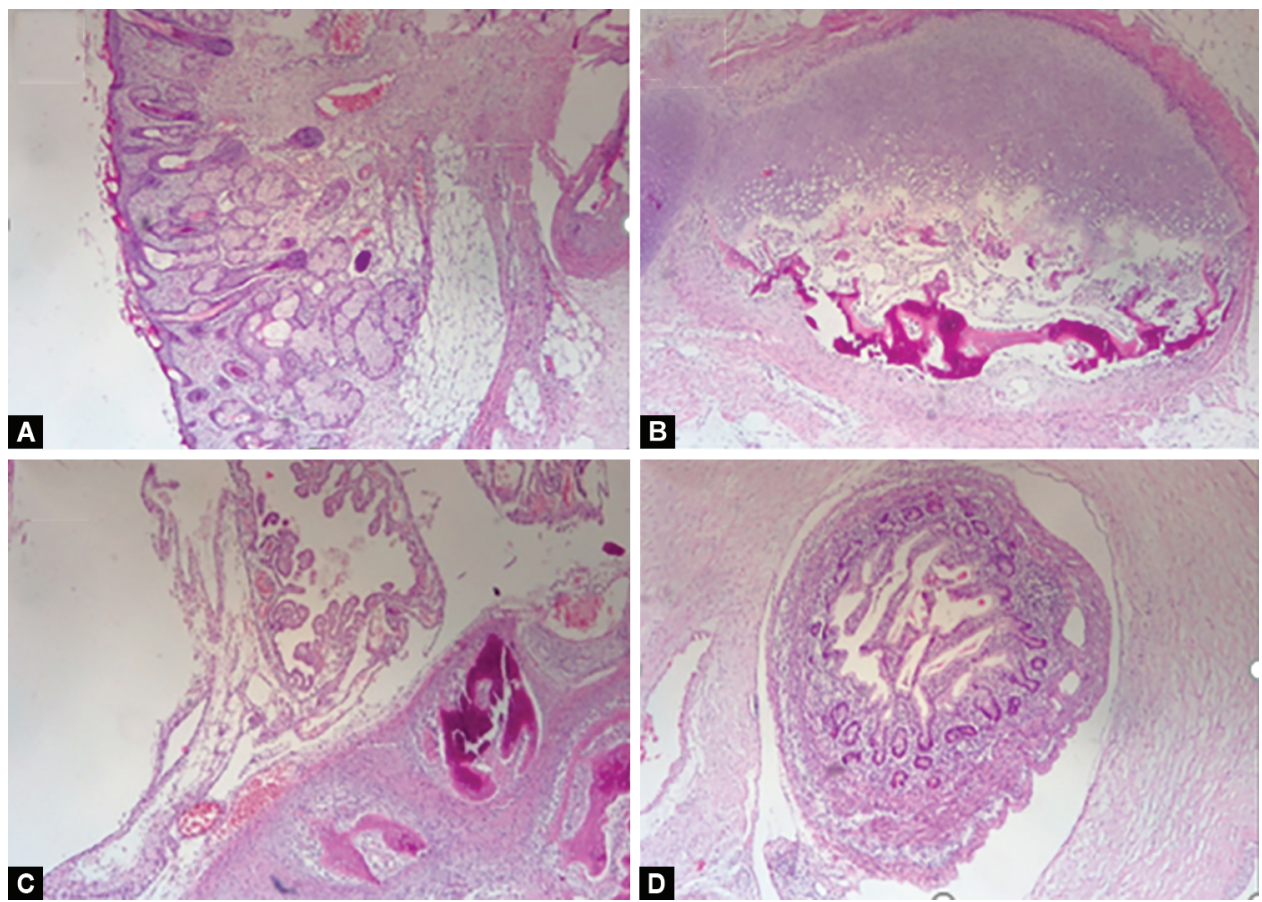

Figs 4A to D: Microscopic findings of mature tissues: skin and skin appendages and adipose tissue (A); Cartilage and bone (B); Cerebral cortex with choroid plexus (C); Gastrointestinal epithelium (D) 


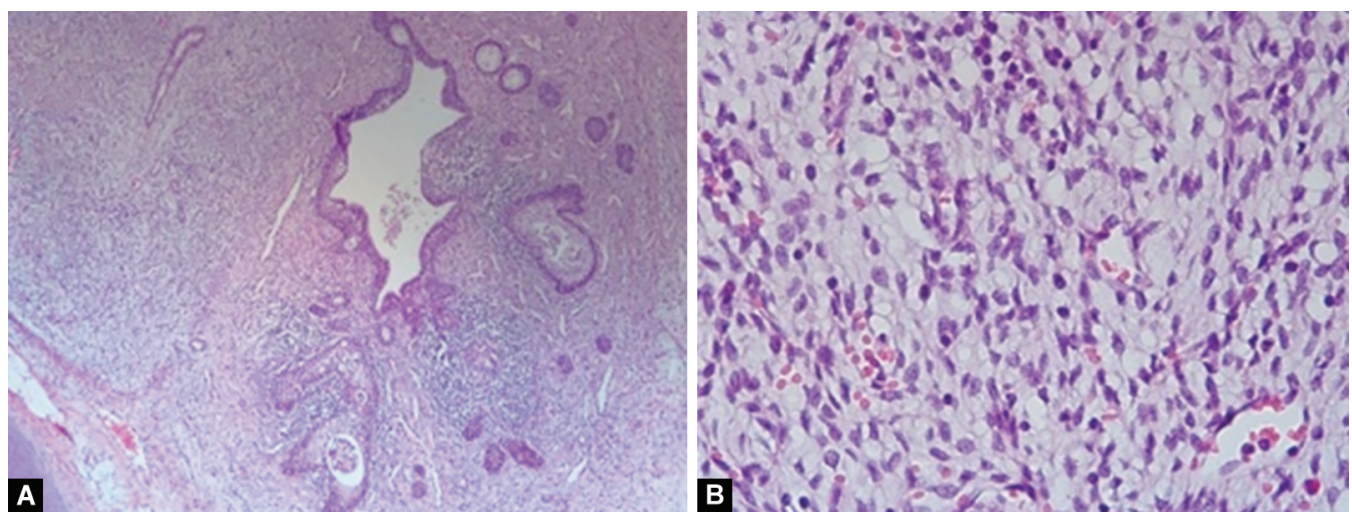

Figs $5 A$ and B: Immature epithelium and mesenchyme (A); Immature mesenchyme $(\times 40)(B)$
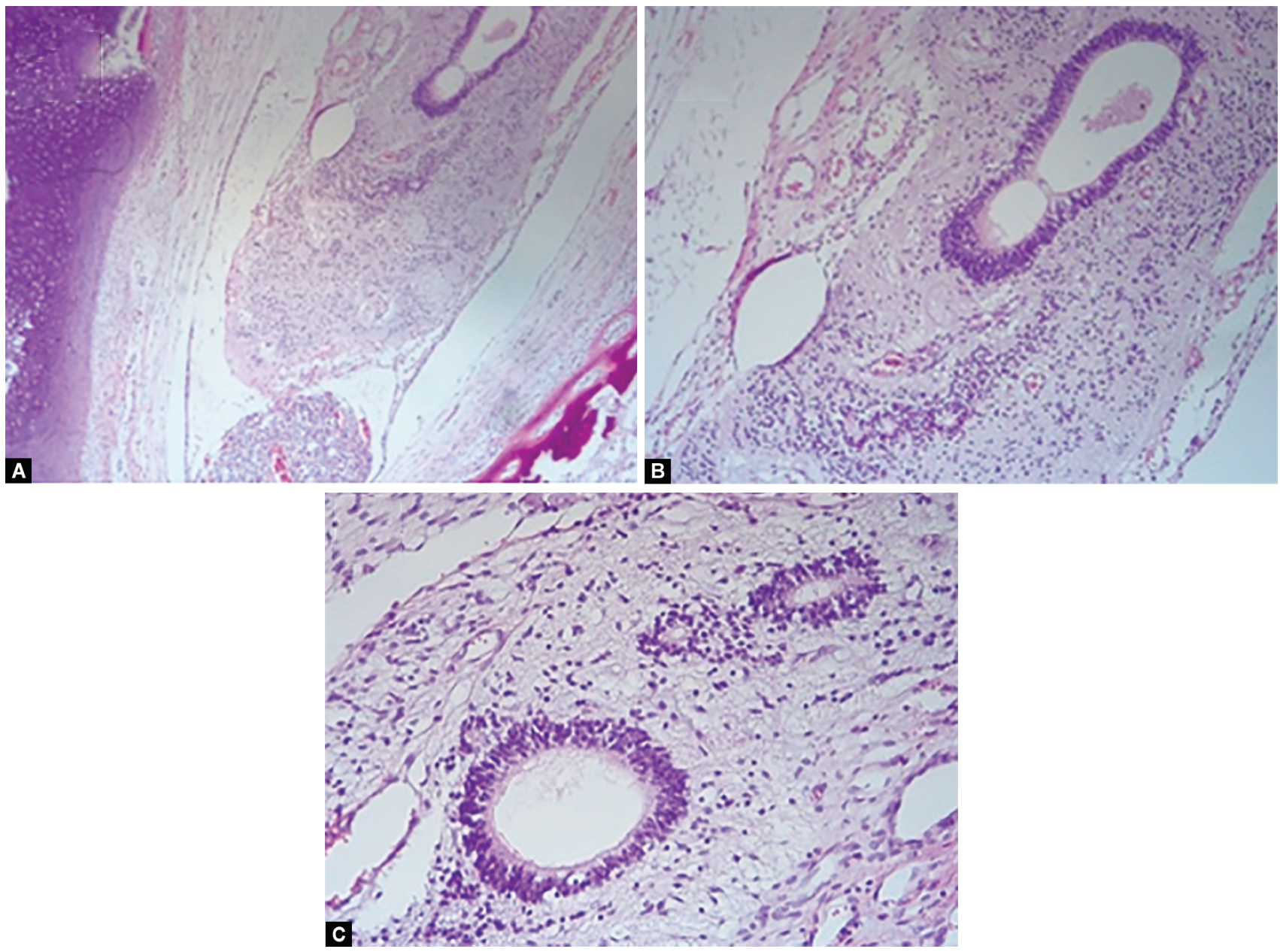

Figs 6 A to C: Neuroectodermal tubules (A); Neuroectodermal tubules $(\times 20)(B)$; Neuroectodermal tubules $(\times 20)(C)$

teratomas, immature teratomas are larger, less well-defined, have a prominent solid component, and are associated with foci of hemorrhage. ${ }^{9}$ Ultrasound differential diagnosis between immature and mature teratomas is difficult with ultrasound characteristics for the presence of multiple cystic and solid areas, a mixture of calcific, fatty, and fluid densities and are associated with a greater number of solid elements than mature teratomas. Based on IOTA pattern recognition visualization of irregular external tumor wall, a lesion with multilocularity, vascularized papillary with grade IV of vascularization score or centrally distributed blood vessel in the mass should raise concern for the possibility of malignancy. ${ }^{6,7}$ Typically younger patients are affected, so preservation of fertility is very important in the management of immature teratomas. For FIGO stage I and grade I tumors, only unilateral oophorectomy is necessary with the preservation of the other ovary. ${ }^{10}$ Laboratory findings like increased levels of alpha-fetoprotein in serum are only in part of the patients with pure immature teratoma. There is a hypothesis that the source of production of elevated levels of alpha-fetoprotein is the neural immature tissue. So the detection of alpha-fetoprotein is not sensitive or specific for the independent 
diagnosis of an immature teratoma. In contrast, ultrasonography in combination with color Doppler is usefull. ${ }^{11}$ The grading system is done by Norris classification and the overall survival rate for stage III disease is 30\%. ${ }^{12}$ Long-term follow-up is very important because studies are showing that recurrent masses can appear many years after the primary tumor. ${ }^{13}$ The incidence of the recurrence of germ cell tumors is $3.4-5.1 \%$, and bilateral or multiple cysts might be a predictive factor of recurrence. Recurrence of immature teratomas is very rare, with a $1 \%$ incidence. The mean time interval until the development of recurrence of mature cystic teratomas is 9 years and for malignant germ cell tumors, it is 2 years. Immature teratoma can be found after previous surgery for mature benign teratoma. ${ }^{14}$ Surgical rupture of the tumor may cause gliomatosis peritonei (implants of mature glial tissue in the peritoneum). Gliomatosis peritonei is relatively rare and not reported to have a poor prognosis when associated with an immature teratoma. ${ }^{15}$

\section{Conclusion}

Immature teratoma is a very rare germ cell tumor with fast growth potential. It is important to make an early diagnosis and proper surgical staging and treatment. $2 \mathrm{D}$ and $3 \mathrm{D}$ vaginal ultrasound examination and analysis of the tumor size, borders, components, and vascularization can help the gynecologist making the right diagnosis. Preservation of fertility is important in younger patients if the tumor is in stage and grade I. Patient should be closely monitored in case of relapse of the tumor.

\section{References}

1. Alwazzan AB, Popowich S, Dean E, et al. Pure immature teratoma of the ovary in adults. thirty-year experience of a single tertiary care center. Int J Gynecol Cancer 2015;25(9):1616-1622. DOI: 10.1097/ IGC.0000000000000541.

2. Smith $\mathrm{HO}$, Berwick $M$, Verschraegen $\mathrm{CF}$, et al. Incidence and survival rates for female malignant germ cell tumors. Obstet Gynecol 2006;107(5):1075-1085. DOI: 10.1097/01.AOG.0000216004.22588.ce.

3. Ihara T, Ohama K, Satoh $\mathrm{H}$, et al. Histologic grade and karyotype of immature teratoma of the ovary. Cancer 1984;54(12):
2988-2994. DOI: 10.1002/1097-0142(19841215)54:12<2988::AIDCNCR2820541229>3.0.CO;2-U.

4. Norris $\mathrm{HJ}$, Zirkin HJ, Benson WL. Immature (malignant) teratoma of the ovary: a clinical and pathologic study of 58 cases. Cancer 1976;37(5): 2359-2372. DOI: 10.1002/1097-0142(197605)37:5<2359::AIDCNCR2820370528>3.0.CO;2-Q.

5. "How is Ovarian Cancer Staged?", American Cancer Society.

6. Timmerman D, Valentin L, Bourne TH, et al. Terms definitions and measurements to describe the sonographic features of adnexal tumors:a consensus opinion from the international ovarian tumor analysis (IOTA) group. Ultrasound Obstet Gynecol 2000;16(5): 500-505. DOI: 10.1046/j.1469-0705.2000.00287.x.

7. Timmerman D, Ameye L, Fischerova D, et al. Simple ultrasound rules to distinguish between benign and malignant adnexal masses before surgery: prospective validation by IOTA group. BMJ 2010;341:c6839. DOI: 10.1136/bmj.c6839Published 2010 Dec 14.

8. Crum CP, Lee KR, Nucci MR, et al. Diagnostic gynecologic and obstetric pathology, Philadelphia, PA: Saunders/Elsevier; 2006. p. 937.

9. Nimkin K, Gupta P, McCauley R, et al. The growing teratoma syndrome. Pediatr Radiol 2004;34(3):259-262. DOI: 10.1007/s00247-0031045-z.

10. Deodhar KK, Suryawanshi P, Shah M, et al. Immature teratoma of the ovary: a clinicopathological study of 28 cases. Indian J Pathol Microbiol 2011;54(4):730-735. DOI: 10.4103/0377-4929.91508.

11. El-Bahrawy M. Alpha-fetoprotein-producing non-germ cell tumours of the female genital tract. Eur J Cancer 2010;46(8):1317-1322. DOI: 10.1016/j.ejca.2010.01.028Epub 2010 Feb 23. PMID: 20185298.

12. Donny JK, Pfeiffer JD, Huettner PC. Ovary. In: Humphrey PA, Dehner LP, Pfeiffer JD, ed. The Washington Manual of Surgical Pathology. 1st ed., Wolter Kluwer(India) Ltd; 2009. p. 39413.

13. Djorghevic B, Euscher D E, Malpica A. Growing teratoma syndrome of the ovary: review of literature and first report of a carcinoid tumor arising in a growing teratoma of the ovary. Am J Surg Pathol 2007;31(12):1913-1918. DOI: 10.1097/PAS.0b013e318073cf44P MID:18043048.

14. Kazurhiro N, Naoto F, Taketoshi N, et al. Immature teratoma after three laparoscopic resection for mature cystic teratomas. Case Rep Obstet Gynecol 2014;2014:264959. DOI: 10.1155/2014/263959.

15. Hamada $Y$, Tanano A, Sato $M$, et al. Ovarian teratoma with gliomatosis peritonei: report of two cases. Surg Today 1998;28(2):223-226. DOI: 10.1007/s005950050112. 